\title{
Erratum: Axion Dark Matter Coupling to Resonant Photons via Magnetic Field [Phys. Rev. Lett. 116, 161804 (2016)]
}

Ben T. McAllister, Stephen R. Parker, and Michael E. Tobar

(Received 28 July 2016; published 5 October 2016)

DOI: 10.1103/PhysRevLett.117.159901

In this erratum we present an error in our recent Letter and an article which comments on our analysis [1]. We derived for the first time the magnetic form factor, which gives a general formula for the magnetic coupling of a photon produced via the inverse Primakoff effect in a Sikivie Haloscope axion dark matter detector. Previously only the electric coupling had been considered. In our Letter, Eqs. (1)-(16) are all correct, and the electromagnetic form factor may be generally written as

$$
\begin{aligned}
C_{\mathrm{EM}} & =\frac{C_{E}+C_{B}}{2} \\
& =\frac{\left|\int d V_{c} \vec{E}_{c} \cdot \overrightarrow{\hat{z}}\right|^{2}}{2 V_{c} \int d V_{c} \epsilon_{r}\left|E_{c}\right|^{2}}+\frac{\frac{\omega_{a}^{2}}{c^{2}}\left|\int d V_{c} \frac{r}{2} \vec{B}_{c} \cdot \overrightarrow{\hat{\phi}}\right|^{2}}{2 V_{c} \int d V_{c} \frac{1}{\mu_{r}}\left|B_{c}\right|^{2}},
\end{aligned}
$$

where $\mu_{r}$ and $\epsilon_{r}$ are the relative permeability and permittivity of the medium within the cavity. However, Eq. (17) in our Letter,

$$
C_{B}=\frac{\frac{\omega_{a}^{2}}{c^{2}}\left|\int d V_{c} B_{c_{\phi}} \frac{r-e \cos \phi}{2}\right|^{2}}{V \int d V_{c}\left|B_{c}\right|^{2}},
$$

which gives an expression for the general magnetic form factor for all $\mathrm{TM}_{0 n 0}$ modes when the cavity is offset from the center of the solenoid, may be shown to be in error by a factor of $r / r_{c}$. The correct result may be found by considering a diagram of the problem (Fig. 1). The cavity magnetic field is in the cavity $\phi_{c}$ direction (and a function of the cavity radius), while the

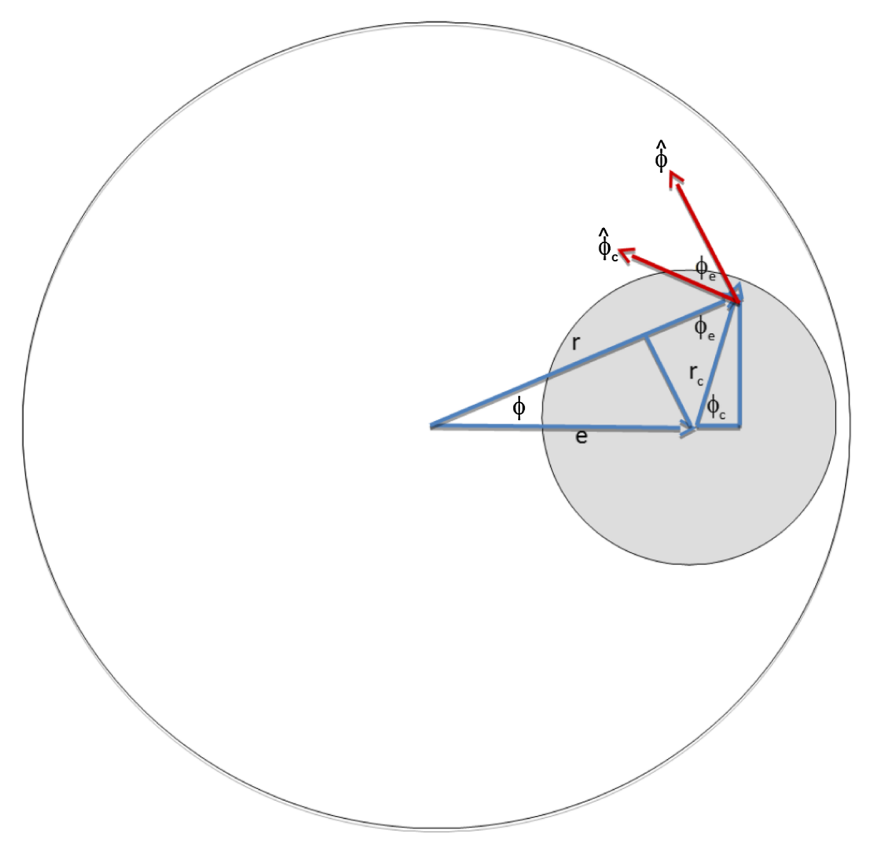

FIG. 1. A diagram of the offset cavity experiment, the unit vectors are shown in red. The angle $\phi_{e}$ is defined as the angle between these unit vectors. All important parameters are labeled. The cavity is shown in grey and solenoid is shown in white. 
axion induced magnetic field is in the solenoid's $\phi$ direction, and proportional to the radial distance in the solenoid to the point of integration. Generally speaking these two directions are not the same, and in the case for a cavity offset by some distance, $e$, from the center of the solenoid the dot product of the $\phi$ and $\phi_{c}$ unit vectors is nontrivial. This is the structure analyzed in our Letter. We define the angle between the two unit vectors as $\phi_{e}$. Since both are unit vectors (of magnitude one) the dot product can be written as

$$
\hat{\phi} \cdot \hat{\phi}_{c}=\cos \phi_{e}
$$

Thus $C_{B}$ becomes

$$
C_{B}=\frac{\frac{\omega_{a}^{2}}{c^{2}}\left|\int d V_{c} B_{c}\left(r_{c}\right) \frac{r}{2} \cos \left(\phi_{e}\right)\right|^{2}}{V \int d V_{c}\left|B_{c}\right|^{2}} .
$$

Here

$$
\cos \phi_{e}=\frac{r-e \cos \phi}{r_{c}}
$$

(which comes from trigonometry as can be seen in Fig. 1). Thus, the integral reduces to

$$
C_{B}=\frac{\frac{\omega_{a}^{2}}{c^{2}}\left|\int d V_{c} B_{c_{\phi}} \frac{r-e \cos \phi}{2} \times \frac{r}{r_{c}}\right|^{2}}{V \int d V_{c}\left|B_{c}\right|^{2}} .
$$

Equation (2) differs from Eq. (1) [Eq. (17) in the Letter] by a factor of $r / r_{c}$. At some point in our analysis, a factor of $r / r_{c}$ has propagated through our numerical calculations, causing an error in the presented results. This changes our conclusion that the form factor varies with offset. When evaluating the form factor with Eq. (2) it remains constant with offset for all $\mathrm{TM}_{0 n 0}$ modes. Meanwhile, a recent comment [1] on our analysis claimed the dot product between these unit vectors to be

$$
\hat{\phi} \cdot \hat{\phi}_{c}=\cos \left(\phi+\phi_{c}\right) .
$$

If we follow through with this expression for $\hat{\phi} \cdot \hat{\phi}_{c}$, we arrive at the following expression for $C_{B}$ :

$$
C_{B}=\frac{\frac{\omega_{a}^{2}}{c^{2}}\left|\int d V_{c} \mathrm{~B}_{c_{\phi}} \frac{r_{c} \cos 2 \phi_{c}+e \cos \phi_{c}}{2}\right|^{2}}{V \int d V_{c}\left|B_{c}\right|^{2}},
$$

which evaluates to zero for all offset values, when integrated over the full cavity limits.

[1] S. Lee, S. W. Youn, and Y. K. Semertzidis, Comment on "Axion Dark Matter Coupling to Resonant Photons via Magnetic Field," arXiv:1606.09504. 